\title{
Risk factors for frequent users of the emergency department among adults aged 55 and older
}

\author{
Deana Hays*1, Barbara Penprase ${ }^{1,2}$, Suha Kridli ${ }^{1}$ \\ ${ }^{1}$ School of Nursing, Oakland University, Rochester, Michigan, United States \\ ${ }^{2}$ Crittenton Hospital Medical Center, Rochester, Michigan, United States
}

Received: March 2, 2018

DOI: $10.5430 /$ jnep.v8n9p96
Accepted: April 22, 2018

URL: https://doi.org/10.5430/jnep.v8n9p96

\begin{abstract}
Introduction: Excessive use of the emergency department (ED) is a major source of healthcare expenditure. ED frequent users, have been identified as a major contributing factor to a disporportionate amount of ED visits and costs, making up $20 \%$ to $30 \%$ of all annual visits. The aim of the study was to identify risk factors that place adults age 55 and older at risk for frequent ED use. Methods: The Transitional Care Model (TCM): Hospital Discharge Screening Criteria for High Risk Older Adults was used to identify risk factors for frequent use of ED services in adults 55 and older.

Results and conclusions: A third of the sample (33\%) had active behavioral and/or psychiatric issues. A majority of the sample $(87 \%)$ had two or more hospitalizations within 6 months of a prior ED visit, and seventy-two percent were hospitalized within thirty days of an Emergency Department visit. Almost $70 \%$ had at least 1 chronic diagnosis of diabetes (41.5\%), heart failure (35.8\%), or COPD (28\%). Most patients were between ages 70-85 years old and risk factors for ED frequent use included 4 or more coexisting health conditions, 6 or more prescription medications, previous hospital admissions, active behavioral and/or psychiatric issues. Identifying older adults at high risk for ED frequent use may provide earlier interventions and less reliance on ED use for care and treatment of chronic disorders.
\end{abstract}

Key Words: ED Frequent users, Transitional care, Frequent flyers, Transitional care model

\section{INTRODUCTION}

With today's rising healthcare costs, it is essential that healthcare systems find ways to reduce overall costs to remain viable. Excessive use of the emergency department (ED) is often identified as a major source of healthcare expenditure. ${ }^{[1]}$ It is estimated that costs for treating patients in the emergency department are 2 to 3 times higher than in other healthcare settings. ${ }^{[2]}$

Significant healthcare dollars go towards treating frequent ED users who visit the ED several times a year, and are often admitted to the hospital. An estimated $\$ 32$ million is spent each year in the United States on unnecessary use of ED services. The cost for frequent users varies considerably and is reported to be up to 15 times higher than the cost for nonfrequent users; nevertheless, the number of ED visits in the United States continues to rise. ${ }^{[3]}$

Frequent users of the ED account for a large portion of visits, up to $34 \%$ annually in the United States. ${ }^{[4-7]}$ Nearly $50 \%$ of Medicare dollars are spent on providing healthcare services to ED frequent users and costs associated with subsequent visits are even higher. ${ }^{[8]}$ Frequent use of Emergency Department services correlates with higher rates of hospital admissions, further driving health care spending. ${ }^{[9-13]}$ After frequent ED use, older adults have been found to be at risk

*Correspondence: Deana Hays; Email: hays@ oakland.edu; Address: School of Nursing, Oakland University, Rochester, Michigan, United States. 
for poor quality of life. ${ }^{[1]}$

How frequent users of the Emergency Department are defined and what places patients at risk for overutilization of Emergency Department services remains unclear. The number of visits that defines an ED frequent user varies among studies; ranging from 3 to $12 \mathrm{ED}$ visits over a 12-month period with little rationale as to why these numbers are chosen. ${ }^{[10,14,15]}$

Past studies have reported risk factors for frequent ED use as patients of low socioeconomic status, those without health insurance and lack of a primary care physician. ${ }^{[16,17]}$ Recent studies, however, have suggested that ED frequent users are more likely to be insured through Medicaid or Medicare and are just as likely as non-frequent users to have a primary care provider. ${ }^{[18,19]}$ ED frequent users have also been found to average twice the number of visits to their primary care provider per year compared to infrequent ED users. ${ }^{[18]}$

Multiple health conditions, chronic illness, and chronic pain have all been found to contribute to ED overutilization, particularly for older adults. ${ }^{[10,19,20]}$ One study ${ }^{[20]}$ found patients having two or more medical conditions increased the overall odds of frequent ED use, adjusted OR = 7.9; 95\% CI (4.6 to 13.4). In a comparison of selected common chronic diseases between frequent and infrequent users, one study noted significant differences in frequency of ED visits for hypertension, sickle cell anemia, and depression $(p=.01, .004$, and .017, respectively). ${ }^{[18]}$ Other investigations have found stroke, congestive heart failure, dementia, asthma, diabetes, and ischemic heart disease to be common chronic illnesses among older adult ED frequent users. ${ }^{[10,20,21]}$

Higher incidences of chronic mental health issues have also been found in ED frequent users compared to non-frequent users. ${ }^{[22]}$ Advanced age has been identified as a major risk factor for overutilization of ED services, with older adults representing the largest age group of ED frequent users. ${ }^{[10,23]}$ Elderly patients that frequently visit the ED have also been found to have longer lengths of stay, higher readmission rates and utilize more resources when compared to any other age group. ${ }^{[10,23-25]}$

In addition, demographic results from the CDC indicate that racial differences are important risk factors for frequent ED use by the elderly. ${ }^{[23]}$ In patients age 65 to 74 years of age, during the period 1997 to 2013, African Americans were shown to be some of the highest users of ED services. Subsequent research indicates that the elderly and African Americans are among the most frequent ED users. ${ }^{[2,26]}$ With these factors considered, the profile of frequent ED users may vary from hospital to hospital, necessitating creative collaborative

Published by Sciedu Press nurse educational programs to promote positive patient care outcomes in this deceptively diverse population.

Older adult patients with co-morbidities have been identified as the highest population of frequent ED users. ${ }^{[10,27]}$ This is often caused by exacerbations of underlying chronic disease. When the patient is treated in the ED, he or she is treated for the admitting chief complaint without addressing other issues that may be related to the patient's co-morbidities. Providers in the ED setting rarely have the resources to educate patients and their caregivers about how to prepare for and treat the numerous medical issues related to having two or more co-morbidities after discharge. There are multiple factors that contribute to ED overutilization among this population. The purpose of this article is to identify specific risk factors for ED frequent use among adults age 55 and older.

\section{MeTHODS}

A descriptive retrospective design was used in this study. A secondary data analyses was conducted by reviewing electronic health records of adult patients with six or more visits to the Emergency Department within a single year period. Subjects were screened for risk factors using the Transitional Care Model (TCM): Hospital Discharge Screening Criteria for High Risk Older Adults. ${ }^{[28]}$

\subsection{Instrumentation}

High-risk screening in the older adult population has been implemented in various settings but few studies have screened for risk factors in frequent users of the Emergency Department. Bixby and Naylor ${ }^{[28]}$ devised a screening tool from several randomized clinical control trials to explore the implementation of a transitional care model for older adults transitioning from hospital to home. The Transitional Care Model (TCM): Hospital Discharge Screening Criteria for High Risk Older Adults screens for high-risk patients in transitions from hospital to home (see Table 1). The presence of 2 or more of 11 screening criteria has been shown to significantly increase the probability of poor outcomes and increased readmission rates following hospital discharge. This tool has not been tested in older adult ED frequent users; however, factors identified in this tool may contribute to patients' repeated utilization of the Emergency Department.

\subsection{Sample and setting}

Data was collected from the electronic health records at a large suburban, Midwestern healthsystem in Michigan. A demographic profile of ED frequent users for this setting was conducted prior to this study. 1,237 patients met the criteria of six or more visits within a 12 month period with greater than $50 \%$ being over the age of $55 .^{[10]}$ Inclusion criteria in- 
cluded adult men and women age 55 and older who had 6 or more ED visits within a single year. Charts with incomplete demographic data were excluded.

Table 1. TCM: Hospital discharge screening criteria for high risk older adults

If "yes" to 2 or more of the following considered high risk:
Age 80 or Older
Moderate or severe functional deficit
An active behavioral and/or psychiatric health issue
Four or more co-existing health conditions
6 or more prescribed medications
Two or more hospitalizations in the past 6 months
A hospitalization within the past 30 days
Inadequate support system
Low health literacy
Documented history of non-adherence to the therapeutic regimen
or
If suspected or confirmed cognitive impairment alone or in
combination with any of the above risk factors

\subsection{Procedure}

Prior to commencement of data collection, Institutional Review Board (IRB) approval was obtained from the hospital IRB board and university IRB. All data were presented as aggregated information, kept strictly confidential and not shared beyond the research team. Data with patient identifiers were immediately removed from the sample once collected from individual records.

\subsection{Data analysis}

A random sample of 200 files was reviewed utilizing the TCM screening tool. The presence of 2 or more of the 11 screening criteria has been shown to significantly increase the probability of hospital readmission and poorer outcomes after hospital discharge. ${ }^{[28-31]}$ The majority of the sample was between ages 70 and 85 years of age.

Risk factors were identified using descriptive statistics to determine the highest frequencies. The data were reported as percentage of total sample for the following variables: 6 or more prescription medications and 2 or more hospitalizations within the past 6 months. Categorical variables were reported in the form of percentages for the following: hospitalization within 30 days, documented behavioral or psychiatric issues, and documented cognitive impairment, all of which were categorized as "present" or "not present". Behavioral/psychiatric conditions were determined using ICD-9 codes for any of the following: depression, personality disorder, substance abuse disorder, anxiety disorder, somatization disorder, and psychotic disorder. Cognitive impairment was determined using the following diagnoses in the nursing or medical documentation: delirium, confusion, and dementia.
In addition, the presence of 1 or more of three chronic illnesses, diabetes, COPD, and heart failure, was reported as a percentage. These three chronic conditions have also been linked to higher 30-day readmission rates and longer inpatient lengths of stay. ${ }^{[32]}$ All data were analyzed using SPSS 19.

\section{RESUltS}

Data from the TCM screening tool revealed $96.5 \%$ of the sample met 2 or more of the criteria. The majority of the sample $(82 \%)$ had 4 or more coexisting health conditions as well as 6 or more prescription medications. A third of the sample (33\%) had active behavioral and/or psychiatric issues. A majority of the sample (87\%) had two or more hospitalizations within 6 months of a prior ED visit, and 72\% were hospitalized within 30 days of an ED visit. A smaller percentage of the sample $(19.5 \%)$ had a documented cognitive impairment. In terms of comorbid conditions, $68.9 \%$ had at least 1 chronic diagnosis of heart failure, diabetes, or COPD, with $39.4 \%$ having just 1 of the 3 chronic conditions, $22.8 \%$ having 2 or more chronic conditions, and $6.7 \%$ having all 3 chronic conditions. The most frequent chronic condition found in this sample was diabetes $(41.5 \%)$, followed by heart failure (35.8\%) and COPD (28\%) (see Table 2).

\section{Discussion}

Although much of the literature has focused on demographic data for ED frequent users, no known studies to date have identified what places older adults at risk for frequent utilization of Emergency Department services. This study examined risk factors found in older adult frequent users of the ED, utilizing a screening tool that identifies older adults at risk for hospital readmission. Six of the 11 risk factors identified in the Transitional Care Model: Hospital Discharge Screening Criteria for High Risk Older Adults were also found in ED frequent users in this study.

As healthcare expenditures for ED visits continue to rise, patients age 65 and older account for the highest consumers of ED resources in the United States. ${ }^{[33]}$ The overall findings from this study support more recent findings in the literature that age and co-existing chronic conditions are risk factors for frequent ED utilization. ${ }^{[10,34]}$ The findings from the present study indicate that older adults who frequently return to the Emergency Department for medical care had at least 1 chronic condition: diabetes, heart failure, or COPD.

Older adults who frequently use ED services have higher readmission rates compared to non-frequent users. ${ }^{[9,11,35,36]}$ Of the ED frequent users in this study, seventy-two percent were admitted within thirty days of a previous ED visit, and eighty-seven percent had at least two hospital admissions 
within a 6-month period from the previous ED visit. Therefore, based on these findings, 30-day readmission from a prior ED visit or 2 hospital admissions within 6 months of a prior ED visit are risk factors for frequent use of the Emergency Department by older adults.

Documented psychiatric or an active behavioral health issues and cognitive impairment were also found to be risk factors in ED frequent users in this study. Although mental health and cognitive impairment were not found to be presenting problems or among the most common ICD-9 codes for ED frequent users in a previous study, ${ }^{[10]}$ the research findings did demonstrate that at least one third of ED frequent users had a co-existing mental health issue or cognitive impairment. Therefore, the findings support co-existing mental health issues and/or cognitive impairment as factors that place older adults at risk for becoming ED frequent users.

Table 2. Results

\begin{tabular}{ll}
\hline High Risk Criteria & Frequency \\
\hline An active behavioral or psychiatric health issue & $33 \%$ \\
Four or more co-existing health conditions & $82 \%$ \\
Six or more prescription medications & $82 \%$ \\
Two or more hospitalizations within the past 6 months & $87 \%$ \\
A hospitalization within 30 days & $72 \%$ \\
Cognitive impairment & $19.5 \%$ \\
Comorbid conditions: diabetes mellitus, heart failure, COPD & $68.9 \%$ \\
1 comorbid condition & $39.4 \%$ \\
2 comorbid conditions & $22.8 \%$ \\
All 3 comorbid conditions & $6.7 \%$ \\
Percentage with diagnosis of diabetes mellitus & $41.5 \%$ \\
Percentage with heart failure & $35.8 \%$ \\
Percentage with COPD & $28 \%$ \\
\hline
\end{tabular}

Overall, ED frequent users $(96.5 \%)$ in this study had at least 2 or more risk factors identified in the TCM screening tool. Early screening of ED frequent users for risk factors can be instrumental in identifying older adults at risk for overutilization of ED resources. Identifying these risk factors is not just key to reducing cost but a means to improve overall health for this population. In fact, frequent users of the Emergency Department have been found to have higher rates of mortality and may be more likely to die during an ED visit or subsequent admission than infrequent users. ${ }^{[9,10]}$ Better screening methods are needed to assist Emergency Departments in improving care of older adults. Screening for risk factors in older adults at risk for frequent utilization of the ED may lead to better health outcomes and overall mortality rates for this population.

\subsection{Nursing implications}

The results of this research have led our nursing school to pilot a transitional care program by these researchers at a local hospital. The program involves nurse practitioner students' clinical experiences for one semester to take place in the Emergency Department. Patients who meet the criteria of age 65 years or older, with two or more comorbidities, and have been admitted to the ED at least three times within the year are asked to participate in the transitional care program. For those patients who agree to participate, the nurse practitioner students under the supervision of a nurse practitioner faculty, follow these same patients for a minimum of sixty days' post discharge from the Emergency Department. The students make an initial home visit within 5 days of discharge and then again at 55 to 60 days' post ED discharge. Home visits and/or phone calls then are done weekly and are determined by the patient's conditions and needs. The nurse practitioner students works closely with the primary care provider during the 60-day period to assure collaborative, coordinated care while the patients are transitioning from hospital to home. Patients are screened for depression, anxiety, and limitations in functional status, nutrition deficiencies, home safety factors, and/or a decline in activities of daily living and re-evaluated at the time of the last home visit for improvements in relation to outcomes and response to prescribed therapies/interventions. Medication reconciliation is also completed. Importantly, we know that once older adults screen positive for these risk factors they will continue to be at risk. ${ }^{[31]}$ The program has been in its pilot stage for one year and data related to the program is being analyzed to 
understand the overall outcome effectiveness.

\subsection{Limitations}

There are several limitations to this study. One major limitation was the use of a single location for conducting this research. Demographic characteristics may vary depending on geographic location. Therefore, generalization of these results to other Emergency Departments is not possible. The researchers have replicated the study at two different hospital sites and are analyzing the data presently. Replication of this research in other geographical locations is needed to identify if these risk factors for frequent ED use are present in other older adults to understand the validity of the frequent ED users profile developed by this research.

The researchers were also not able to determine if one or more of three chronic conditions, diabetes, COPD, and heart failure, were directly related to ED visits. Presenting complaints and discharge ICD-9 codes did not always reflect the presence of chronic illness as being related to an ED visit for older adults. A larger sample size may find other chronic conditions present in older adult ED frequent users.

\section{Conclusion}

Although much research has been published on ED frequent users, limited research focuses specifically on identifying those at risk for frequent ED use and interventions to improve outcomes in this population. Educational programs for ED nurses need to focus on identification of such risk factors and implementation of evidence based geriatric knowledge into practice with attention to management and care coordination of older adults with chronic illness. Screening tools that provide built alerts within the Electronic Health Record could aid the ED nurse in the identification of such risk factors. ${ }^{[37]}$ A nurse led Transitional Care team that includes ED nurses also needs to be investigated as a model for reducing frequent ED visits in high risk older adults.

\section{CONFLicts OF InTEREST Disclosure}

The authors declare that there is no conflict of interest.

\section{REFERENCES}

[1] McWilliams A, Tapp H, Barker J, et al. Cost analysis of the use of emergency departments f or primary care services in Charlotte North Carolina. NC Med J. 2011; 72(4): 265-71.

[2] Wajenburg A, Hwang U, Torres L, et al. Characteristics of frequent geriatric users of an urban emergency department. J Emerg Med. 2011; 43(2): 376-81

[3] South Carolina Institute of Medicine \& Public Health (SCPHI). Report of Frequent Users of Hospital Emergency Departments in South Carolina. 2011.

[4] Capp R, Rosenthal M, Desai M, et al. Characteristics of Medicaid enrollees with frequent ED use. American Journal of Emergency Medicine. 2013; 31(9): 1333-1337. PMid:23850143 https : //doi.org/10.1016/j.ajem.2013.05.050

[5] Hardie T, Polek C, Wheeler E, et al. Characterizing emergency department high-frequent users in a rural hospital. Emergency Medicine. 2015; 32(1): 21-25.

[6] LaCalle E, Rabin E. Frequent users of emergency departments: the myths, the data, and the policy implications. Ann Emerg Med. 2010; 56: 42-48. PMid:20346540 https ://doi.org/10.1016/j.anne mergmed.2010.01.032

[7] Wong J, Marr P, Kwan D, et al. Identification of inappropriate medication use in elderly patients with frequent emergency department visits. Canadian Pharmacists Journal. 2014; 147(4): 248-256. PMid:25360151 https://doi .org/10.1177/17151635145365 22

[8] Kumar G, Klein R. Effectiveness of Case Management Strategies in Reducing Emergency Department Visits in Frequent User Patient Populations: A Systematic Review. Journal of Emergency Medicine. 2013; 44(3): 717-729. PMid:23200765 https://doi.org/10.1 $016 / \mathrm{j} \cdot \mathrm{jemermed} .2012 .08 .035$
[9] Fuda KK, Immekus R. Frequent users of Massachusetts emergency departments: a statewide analysis. Ann Emerg Med. 2006; 48: 9-16. PMid:16781915

[10] Penprase B, Hays D. A Profile for Frequent Emergency Department Users: Determining a Baseline for Targeted Interventions. American Research Journal of Nursing. 2015; 1(2): 22-26.

[11] Roberts DC, McKay MP, Shaffer A. Increasing rates of emergency department visits for elderly patients in the United States, 1993 to 2003. Ann Emerg Med. 2008; 51(6): 769-74. PMid:18069088 https ://doi.org/10.1016/j.annemergmed.2007.09.011

[12] Blank FS, Li H, Henneman PL, et al. A descriptive study of heavy emergency department users at an academic emergency department reveals heavy ED users have better access to care than average users. J Emerg Nurs. 2005; 31: 139-44. PMid:15834378 https://doi.org/10.1016/j.jen.2005.02.008

[13] Simonet D. Cost reduction strategies for emergency services: Insurance role, practice changes, and patients' accountability. Health Care Analysis. 2008; 17: 1-19. PMid:18306043 https://doi.org/10 $.1007 / \mathrm{s} 10728-008-0081-0$

[14] Hunt KA, Weber EJ, Showstack JA, et al. Characteristics of frequent users of emergency departments. Am Coll Emerg Phys. 2006; 48(1): $1-8$.

[15] Moore L, Deehan A, Seed P, et al. Characteristics of frequent attenders in an emergency department: analysis of 1-year attendance data. Emerg Med J. 2009; 26: 263-267. PMid:19307386 https://doi.org/10.1136/emj . 2008.059428

[16] Baker D, Stevens C, Brooke R. Regular source of ambulatory care and medical care utilization by patient presenting to a public health emergency department. JAMA. 1994; 271: 1902-1912. PMid:8201724

[17] Pane GA, Farner MC, Salness KC. Health care access problems of medically indigent walk-in patients. Annals of Emergency of Medicine. 1991; 20: 730-733. https ://doi.org/10.1016/S019 6-0644 (05) 80832-7 
[18] Sandoval E, Smith S, Walter J, et al. A comparison of frequent and infrequent visitors to an urban emergency department. J Emerg Med. 2010; 38(2): 115-121. PMid:18462906 https://doi.org/10.1 016/j.jemermed.2007.09.042

[19] Vinton D, Capp R, Rooks S, et al. Frequent Users of US Emergency Departments. Emerg Med J. 2014; 31(7): 526-532. PMid:24473411 https://doi.org/10.1136/emermed-2013-202407

[20] Bieler G, Paroz S, Faouzi M, et al. Social and medical vulnerability factors of emergency department frequent users in a universal health insurance system. Acad Emerg Med. 2012; 19(1): 63-8. PMid:22221292 https://doi.org/10.1111/j.1553-2712.20 $11.01246 . x$

[21] Horney C, Schmader K, Sanders LL. Healthcare utilization before and after an outpatient ED visit in older people. Am J of Emerg Med. 2012; 30(1): 135-142. PMid:21216555 https://doi.org/10.1 016/j.ajem.2010.10.036

[22] Choi NG, DiNitto DM, Marti CN, et al. Association of mental health and substance abuse disorders with presenting problems and outcomes in older adult's emergency department visits. Academic Emerg Med. 2015; 22(11): 1316-1326. PMid:26473592 https://doi.org/10.1111/acem. 12803

[23] CDC. Emergency department visits within the past 12 months by selected characteristics: United States, selected years 1997-2013 (Table 80). 2015.

[24] Gruneir A, Rochon P. Review: emergency department use by older adults: A literature review on trends, appropriateness, and consequences of unmet healthcare needs. Medical Care Research and Review. 2011; 68(2): 131-55. PMid:20829235 https://doi.org/ 10.1177/1077558710379422

[25] Doupe MB, Palanick W, Day S, et al. Frequent users of the emergency departments: Developing standard definitions and defining prominent risk factors. Am Coll Emerg Phys. 2012; 60(1): 24-32.

[26] Milani SA, Crooke H, Cottler LB, et al. Sex difference in frequent ED use among those with multimorbid chronic diseases. Am J Emerg Med. 2016; 34(11): 2127-2131. PMid:27592724 https://doi.org/10.1016/j.ajem.2016.07.059

[27] Billings J, Raven M. Dispelling an urban legend: frequent emergency department users have substantial disease of burden. Health Affairs. 2013; 13(12): 2099-108.
[28] Bixby MB, Naylor MD. The transitional care model: hospital discharge screening criteria for high risk older adults. Try This: Best Practices in Nursing Care to Older Adults. 2009; 26.

[29] Naylor M, Brooten D, Jones R, et al. Comprehensive discharge planning for the hospitalized elderly: a randomized clinical trial. Ann Int Med. 1994; 120(12): 999-1006. https://doi.org/10.7326/00 03-4819-120-12-199406150-00005

[30] Naylor M, Brooten D, Cambell R, et al. Comprehensive discharge planning and home follow-up of hospitalized elders: a randomized control trial. JAMA. 1999; 281(7): 613-620. https://doi.org/ $10.1001 /$ jama. 281.7 .613

[31] Naylor M, Brooten D, Cambell R, et al. Transitional care model for older adults hospitalized with heart failure: 1 randomized clinical trial. J Am Geriatric Soc. 2004; 52(5): 675-84. PMid:15086645 https://doi.org/10.1111/j.1532-5415.2004.52202.x

[32] Kanel K, Elster S, Vrbin C. PRHI readmissions briefs: overview of six target chronic diseases. Pittsburg Reg. Health Init. 2010.

[33] McCraig L, Burt C. CDC national hospital ambulatory medical care survey: 2003 emergency department summary. Advance Data: From Vital and Health Statistics. 2005; 358: 1-40.

[34] Samaras N, Chevalley T, Samaras D, et al. Older adults in the emergency department: A review. Ann Emerg Med. 2010; 56(3): 2619. PMid:20619500 https://doi.org/10.1016/j. annemergme d. 2010.04 .015

[35] Grief CL. Patterns of ED use and perceptions of the elderly regarding their emergency care: a synthesis of recent research. J Emerg Nurs. 2003; 29: 122-6. https://doi.org/10.1067/men.2003.65

[36] McCuster J, Verdon J, Veillette N, et al. Standardized screening and assessment of older emergency department patients: A survey of implementation in Quebec. Can J Aging. 2007; 26(1): 49-57. https://doi.org/10.3138/G236-P856-815W-3863

[37] Ota K, Lazkani M, Orme G. An automated alert system in a transitional care program to improve continuity of care in the emergency department: A strategy for reducing rehospitalizations. J of Emerg Med. 2014; 47(2): 213-214. PMid:24560013 https: //doi.org/10.1016/j.jemermed.2013.11.091 